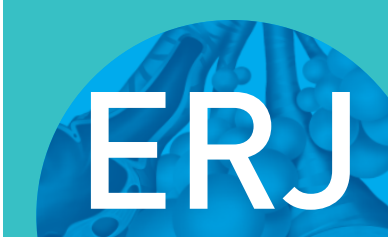

open research

\title{
Xpert MTB/RIF performance to diagnose tuberculosis and rifampicin resistance in a reference centre in southern Brazil
}

\author{
Cinara Silva Feliciano [1] , Lucas José Bazzo Menon", Livia Maria Pala Anselmo, \\ Anzaan Dippenaar ${ }^{2}$, Robin Mark Warren², Wilson Araújo Silva Jr $\mathbb{1}^{3,4}$ and \\ Valdes Roberto Bollela ${ }^{1}$
}

Affiliations: ${ }^{1}$ Dept of Internal Medicine, Ribeirão Preto Medical School, University of São Paulo (FMRP-USP), São Paulo, Brazil. ${ }^{2}$ DST-NRF Centre of Excellence for Biomedical Tuberculosis Research, SAMRC Centre for Tuberculosis Research, Division of Molecular Biology and Human Genetics, Faculty of Medicine and Health Sciences, Stellenbosch University, Stellenbosch, South Africa. ${ }^{3}$ Center for Medical Genomics, Clinics Hospital at Ribeirão Preto Medical School, FMRP-USP, São Paulo, Brazil. "Dept of Genetics, Ribeirão Preto Medical School, FMRP-USP, São Paulo, Brazil.

Correspondence: Valdes Roberto Bollela, Ribeirão Preto Medical School, FMRP-USP, Avenida Bandeirantes 3900, Monte Alegre, CEP: 14049, Ribeirão Preto, São Paulo, Brazil. E-mail: vbollelalafmrp.usp.br

ABSTRACT Effective treatment of tuberculosis (TB) remains a serious public health problem in many countries, including Brazil, especially when considering drug-resistant disease. Xpert MTB/RIF has been implemented in many countries to reduce the time to TB diagnosis and to rapidly detect rifampicin resistance. The study aimed to describe and evaluate Xpert MTB/RIF performance in diagnosing pulmonary TB and rifampicin resistance in a tertiary healthcare facility in Brazil.

A cross-sectional study was performed, which included all isolates of confirmed pulmonary TB patients from 2015 to 2018. Both Xpert MTB/RIF and GenoType MTBDRplus assays were performed to detect rifampicin and isoniazid resistance. In addition, isolates with detected resistance to rifampicin and/or isoniazid were analysed by phenotypic testing using MGIT-960 SIRE kit and whole-genome sequencing (WGS) using Illumina MiSeq Sequencing System.

2148 respiratory specimens tested with Xpert MTB/RIF were included: $\mathrm{n}=1556$ sputum, $\mathrm{n}=348$ bronchoalveolar lavage and $n=244$ gastric washing. The overall Xpert MTB/RIF sensitivity in sputum was $94 \%$ and the overall specificity was $98 \%$. The negative predictive value in sputum of all the patients was $99 \%$ with a positive predictive value of $89 \%$. The concordance between Xpert MTB/RIF and phenotypic susceptibility test was $94.1 \%$, while its concordance with WGS was $78.9 \%$.

Xpert MTB/RIF is a rapid and accurate diagnostic strategy for pulmonary TB, which can contribute to improvement in TB control. However, detection of rifampicin resistance might be associated with falsepositive results.

@ERSpublications

Xpert MTB/RIF has the potential to reduce the time to diagnose TB, with high accuracy, including paucibacillary disease. It is also feasible to detect rifampicin resistance, with a high concordance with phenotypic tests and whole-genome sequencing. http://bit.ly/2WW4jmt

Cite this article as: Feliciano CS, Menon LJB, Anselmo LMP, et al. Xpert MTB/RIF performance to diagnose tuberculosis and rifampicin resistance in a reference centre in southern Brazil. ERJ Open Res 2019; 5: 00043-2019 [https://doi.org/10.1183/23120541.00043-2019].

Received: Feb 152019 | Accepted after revision: June 072019

Copyright $\odot$ ERS 2019. This article is open access and distributed under the terms of the Creative Commons Attribution Non-Commercial Licence 4.0. 


\section{Introduction}

Tuberculosis (TB) remains among the top 10 causes of death worldwide and the leading cause from a single infectious agent. An estimated 10 million new cases and 1.6 million TB-related deaths occurred in 2017, according to a World Health Organization (WHO) report [1].

Drug-resistant TB remains a public health threat, since 558000 rifampicin-resistant cases were estimated in 2017 and of these $82 \%$ were classified as multidrug-resistant (MDR) (resistance to rifampicin and isoniazid). Conventional culture-based drug susceptibility testing is time consuming, which negatively impacts on the transmission of drug-resistant TB in the community [2].

Allied to the strategies to control the disease, in 2011 the WHO endorsed the use of Xpert MTB/RIF (Cepheid, Sunnyvale, CA, USA), which is a rapid, automated and cartridge-based molecular test, as a method for the initial diagnosis test for suspected TB cases. In a timeframe of $2 \mathrm{~h}$, the test detects Mycobacterium tuberculosis DNA and the main mutations that cause rifampicin resistance, providing high sensitivity and specificity values for pulmonary specimens [3]. In addition, the WHO has endorsed Xpert MTB/RIF for use in extrapulmonary specimens, with lower and variable sensitivity values [4].

The Brazilian National Program for TB Control (Ministry of Health) implemented this diagnostic technology in 92 high-burden cities between 2014 and 2015. However, there is very little published information about Xpert performance for pulmonary tuberculosis (PTB) and drug-resistant TB diagnosis in Brazil. Clinics Hospital at the University of São Paulo (Ribeirão Preto, Brazil) has been using Xpert MTB/RIF since 2015 to test both pulmonary and extrapulmonary samples [5].

This study aims to describe and evaluate Xpert MTB/RIF performance in respiratory samples (sputum, bronchoalveolar lavage (BAL), gastric washing) to diagnose PTB and rifampicin resistance in a tertiary reference hospital.

\section{Methods}

This retrospective cross-sectional study enrolled all samples of confirmed PTB patients from 2015 to 2018 in a tertiary hospital located in São Paulo state, southern Brazil. All the individuals who had at least one pulmonary sample tested with Xpert MTB/RIF in the hospital were included. Xpert MTB/RIF was performed according to manufacturer's instructions. All included cases were classified into one of two categories: confirmed PTB and probable PTB cases. In addition to clinical, epidemiological and radiological evaluation, PTB confirmed cases always had a $M$. tuberculosis-positive culture and/or biopsy showing a typical histopathological pattern characterised as a chronic granulomatous inflammatory reaction with caseous necrosis, and the presence of acid-fast bacilli (AFB). Probable cases included those with clinical, epidemiological and radiological evaluation with clinical response to specific treatment to TB [6].

Concentrated smears were stained using Ziehl-Neelsen staining for detection of AFB, and culture was performed in liquid media (BACTEC MGIT 960). M. tuberculosis complex strains were identified in culture using the rapid immunochromatographic test Sd Bioline TBAgMPT64 (Standard Diagnostics, Seoul, South Korea).

In addition, all the isolates were analysed according to manufacturer's instructions using the genotypic test GenoType $^{\circ}$ MTBDRplus (Hain Lifescience, Nehren, Germany), which provides information about mutations associated with rifampicin and isoniazid resistance. All the M. tuberculosis with resistance detected using Xpert ${ }^{\oplus} \mathrm{MTB} / \mathrm{RIF}$ underwent routine phenotypic drug susceptibility tests (DST) on liquid media MGIT-960 SIRE kit (MGIT-960; Becton Dickinson Diagnostic Systems, Sparks, MD, USA). The critical concentration used was $1.0 \mu \mathrm{g} \cdot \mathrm{mL}^{-1}$ for rifampicin and $0.1 \mu \mathrm{g} \cdot \mathrm{mL}^{-1}$ for isoniazid.

Isolates with at least one commercial molecular test indicating rifampicin drug resistance were evaluated using a whole-genome sequencing (WGS) technique. The WGS was performed using an Illumina MiSeq Sequencing System MiSeqV2-500 cycles (Illumina, San Diego, CA, USA). DNA libraries were prepared using the Nextera XT library preparation kit. Sequencing was performed using the MiSeq Reagent Kit v2 (500 cycles) per the manufacturer's protocol, producing 250 bp paired-end reads. TB profiler was used to identify mutations known to cause drug resistance [7]. In addition, sequences were analysed with a pipeline composed of open source software as described previously [8]. Briefly, the trimmomatic tool was used for trimming of adapters and low-quality bases (Phred quality score <20) and filtering for a minimum read length of 36 [9]. Reads were aligned to the M. tuberculosis H37Rv (Genbank: AL123456) genome using three different alignment algorithms: the Burrows-Wheeler alignment tool [10], Novoalign and SMALT [11]. For all sequenced isolates, $>98 \%$ of the reference genome was covered by at least one read and an average depth of coverage of $44(\min 20, \max 80)$ was achieved. The alignment files were subjected to local realignment and de-duplication using the Genome Analysis Toolkit (GATK) [12] and Picard tools [13]. Variants (single nucleotide polymorphisms) and insertion/deletions in coding and 
noncoding regions were identified from each alignment file using GATK [12] and SamTools, and the variants identified in all three alignments were used for further analysis. Variants were annotated and drug resistance was inferred from a combination of drug-resistance mutation libraries [7].

We further evaluated patient records with discrepant results on DST to better define the most likely diagnoses. Xpert MTB/RIF sensitivity, specificity, positive and negative predictive values were calculated using culture results, clinical data and response to specific therapy as the standard for a confirmed TB case.

This study was approved by the Clinics Hospital ethics committee (protocol number: 17471/2014).

\section{Results}

During the study period 2241 respiratory specimens were tested using Xpert MTB/RIF: n=1625 sputum, $\mathrm{n}=354 \mathrm{BAL}$ and $\mathrm{n}=262$ gastric washing. 2148 valid Xpert MTB/RIF results were obtained.

\section{Sputum}

Of the 1625 sputum samples, $69(4.2 \%)$ were excluded due to invalid results and 207 (13.3\%) had a positive Xpert MTB/RIF result. 185 (89.3\%) patients with a positive Xpert MTB/RIF had confirmed TB, of which 178 (96.2\%) were culture-positive. Among three patients with negative culture and positive Xpert MTB/RIF and AFB there were two cases with $M$. tuberculosis detected in culture of nonrespiratory specimens in the same period. One patient had symptoms and chest radiological images suggested TB and cured after TB treatment only.

There were 26 patients with negative culture and AFB with a positive Xpert MTB/RIF result. Four showed symptoms and chest radiological findings and were treated for TB with complete recovery after 6 months. Among the remaining 22 patients, 19 had confirmed previous TB (1-3 years prior), but without detection of active TB during the study period, and three patients had an alternative diagnosis (fungal disease).

Of the 1349 Xpert MTB/RIF negative patients, 10 were culture-positive for M. tuberculosis, 129 were culture-positive for nontuberculous mycobacteria (NTM) and all the others had negative culture for mycobacteria. All six samples with positive AFB and negative Xpert MTB/RIF were identified as NTM. Among the three cases with negative culture and a negative Xpert MTB/RIF but AFB-positive, two had symptoms and chest radiological images suggestive of TB. These patients received treatment for TB and recovered from the symptoms completely. The symptoms of the third patient could be explained by other diagnoses (figure 1).

The overall sensitivity in sputum was 94\%: 98.3\% among AFB-positive samples and 87.2\% for paucibacillary PTB. Overall specificity was 98\%: 100\% among AFB-positive samples and 98.4\% among AFB-negative samples (table 1).

\section{BAL}

Among the 354 BAL samples, six (1.7\%) were excluded due to invalid Xpert MTB/RIF results and 27 (7.8\%) of the 348 valid tests had Xpert-positive results. $22(81.4 \%)$ patients with Xpert-positive results had confirmed TB; 19 out of 22 had positive culture. Among the three cases with negative culture and positive Xpert MTB/RIF and AFB, one patient had M. tuberculosis culture-positive sputum in the same period. The other two patients had confirmed previous TB (1 year prior), but without active disease during the study period.

Five patients were culture-negative, AFB-negative and had a positive Xpert result. Among these, two had $M$. tuberculosis detected in sputum culture in the same period. Two patients had confirmed previous TB (1-2 years prior) without active disease, and the one patient had an alternative diagnosis (false-positive). Overall, among the confirmed TB cases and Xpert-positive, 11 (50\%) were AFB-positive.

Among the 321 negative Xpert cases, four had positive culture results. Three of these were identified as NTM (figure 2).

The sensitivity, specificity, negative predictive value (NPV) and positive predictive value (PPV) for BAL are described in table 1.

\section{Gastric washing}

Among the 262 gastric washing samples, 18 (6.9\%) were excluded due to invalid results. 29 (11.9\%) of the 244 valid tests had an Xpert-positive result and all of these had confirmed TB, 24 with positive culture. Among the Xpert-positive results, 18 (62\%) were AFB-positive. All of the five culture-negative patients had M. tuberculosis detected in a sputum culture taken during the same period. 


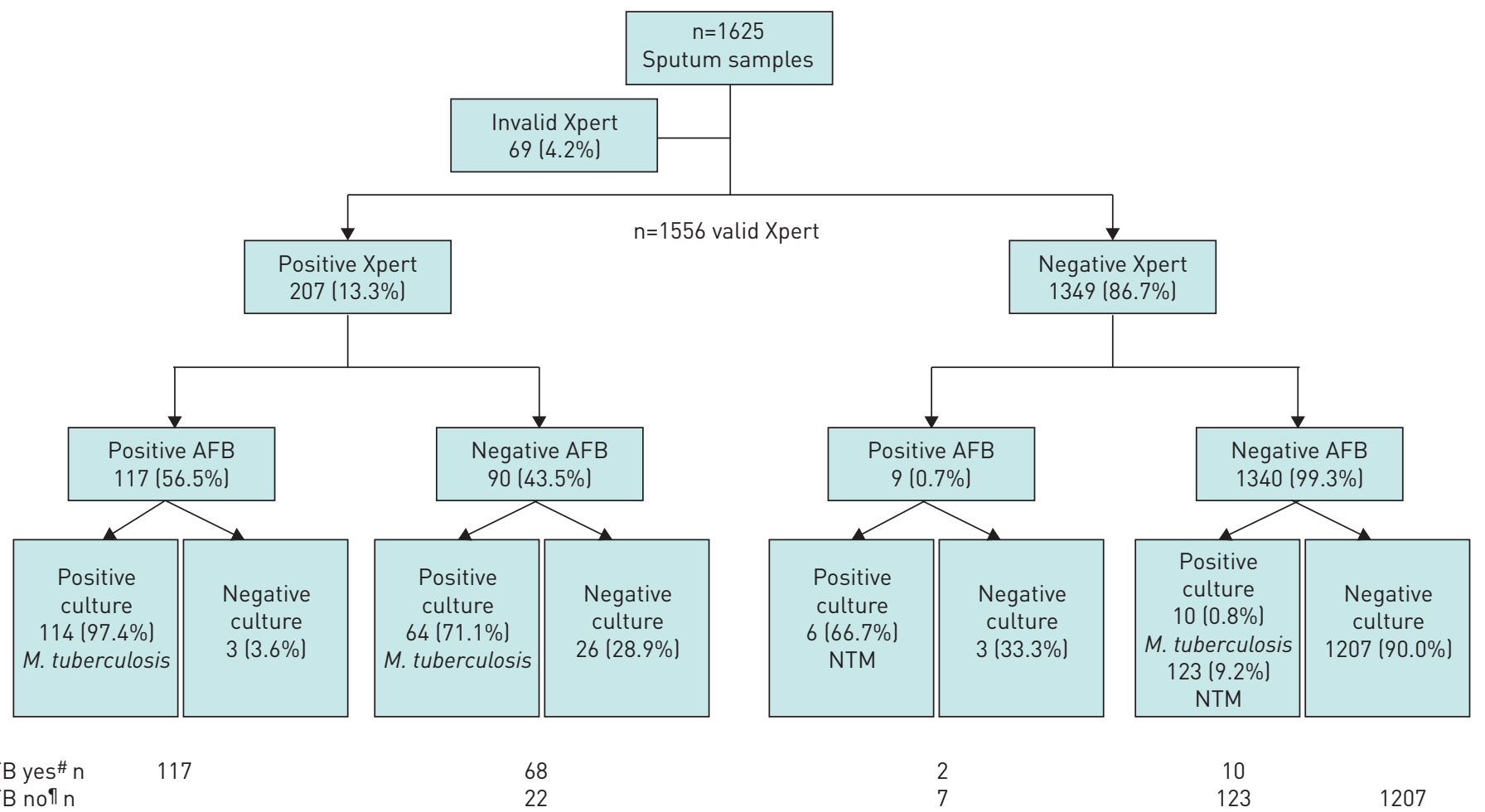

TB yes\# $n$

22

2
7

10

FIGURE 1 Xpert MTB/RIF results for 1625 sputum samples from suspected pulmonary tuberculosis (TB) patients. Xpert: Xpert MTB/RIF; AFB:

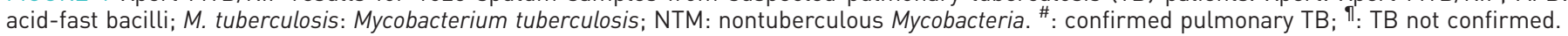

Among the 215 negative Xpert MTB/RIF cases, 11 had positive culture results. 10 out of 11 were identified as NTM and one as M. tuberculosis (figure 3).

The sensitivity, specificity, NPV and PPV for gastric washing are described in table 1.

Table 1 shows Xpert MTB/RIF assay sensitivity, specificity, PPV and NPV for the diagnosis of 2148 respiratory specimens from patients suspected of pulmonary tuberculosis.

Rifampicin-resistance evaluation

Xpert MTB/RIF detected rifampicin resistance in 23 patients (18 in sputum samples, three in BAL and two in gastric washing). 14 (60.9\%) patients had results from at least two Xpert tests, the second one

\begin{tabular}{|c|c|c|c|c|c|c|}
\hline & \multicolumn{2}{|c|}{ Sputum } & \multicolumn{2}{|r|}{ BAL } & \multicolumn{2}{|c|}{ Gastric washing } \\
\hline $\begin{array}{l}\text { Subjects n } \\
\text { Xpert }\end{array}$ & & 56 & & 348 & & 244 \\
\hline Sensitivity & 185/197 & 94 (0.90-0.97) & $22 / 23$ & $96(0.78-1.00)$ & $29 / 30$ & $97(0.83-1.00)$ \\
\hline Specificity & $1337 / 1359$ & $98(0.98-0.99)$ & $320 / 325$ & $98(0.96-0.99)$ & $214 / 214$ & $100(0.98-1.00)$ \\
\hline PPV & $185 / 207$ & 89 (0.84-0.93) & $22 / 27$ & $81(0.62-0.94)$ & $29 / 29$ & $100(0.88-1.00)$ \\
\hline NPV & $1337 / 1349$ & 99 (0.98-1.00) & $320 / 321$ & 99 (0.98-1.00) & $214 / 215$ & 99 (0.97-1.00) \\
\hline \multicolumn{7}{|l|}{ AFB } \\
\hline Sensitivity & 119/197 & $60(0.53-0.67)$ & $9 / 23$ & $39(0.20-0.61)$ & $18 / 30$ & $60(0.41-0.77)$ \\
\hline Specificity & $1352 / 1359$ & 99 (0.99-1.00) & $323 / 325$ & 99 (0.98-1.00) & $214 / 214$ & $100(0.98-1.00)$ \\
\hline PPV & $119 / 126$ & 94 (0.89-0.98) & 9/11 & $82(0.48-0.98)$ & 18/18 & $100(0.81-1.00)$ \\
\hline NPV & $1352 / 1430$ & $95(0.93-0.96)$ & $323 / 337$ & 96 (0.93-0.98) & $214 / 226$ & $95(0.91-0.97)$ \\
\hline
\end{tabular}




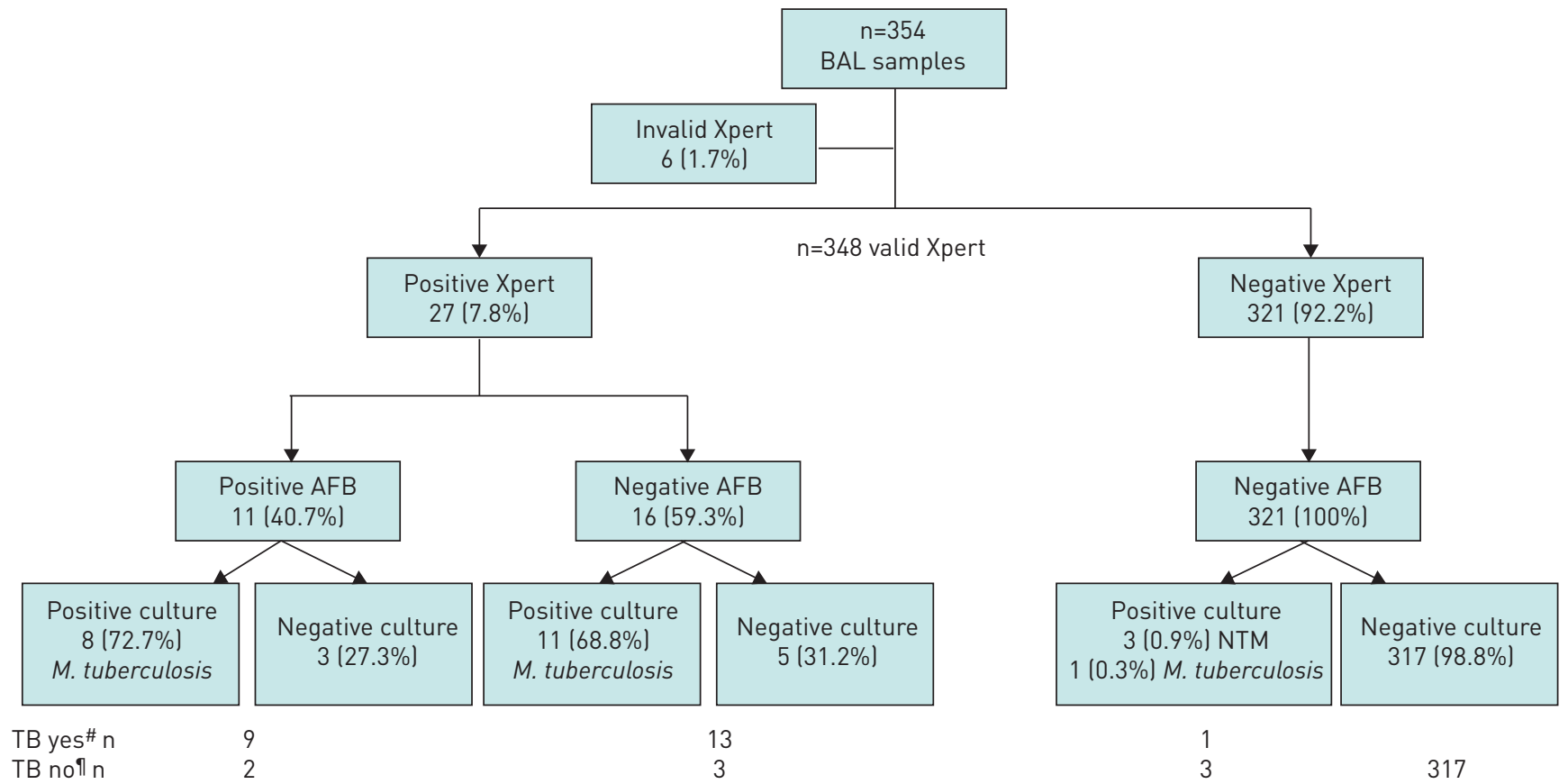

FIGURE 2 Xpert MTB/RIF results for 354 bronchoalveolar specimens from patients suspected of pulmonary tuberculosis (TB). BAL: bronchoalveolar lavage; Xpert: Xpert MTB/RIF; AFB: acid-fast bacilli; M. tuberculosis: Mycobacterium tuberculosis; NTM: nontuberculous Mycobacteria. ${ }^{\#}$ : confirmed pulmonary TB; ๆ: TB not confirmed.

performed after the first result had shown rifampicin resistance. The isolates were tested using Genotype MTBDRplus and only the first sample was submitted to phenotypic DST and WGS. 12 (85.7\%) out of 14 patients had concordant Xpert rifampicin-resistance results and two (14.3\%) had discordant tests (one showing rifampicin resistance and the other with susceptibility to this drug). One of these came from BAL and the other from gastric washing. Nine patients had only one Xpert test with a rifampicin-resistant

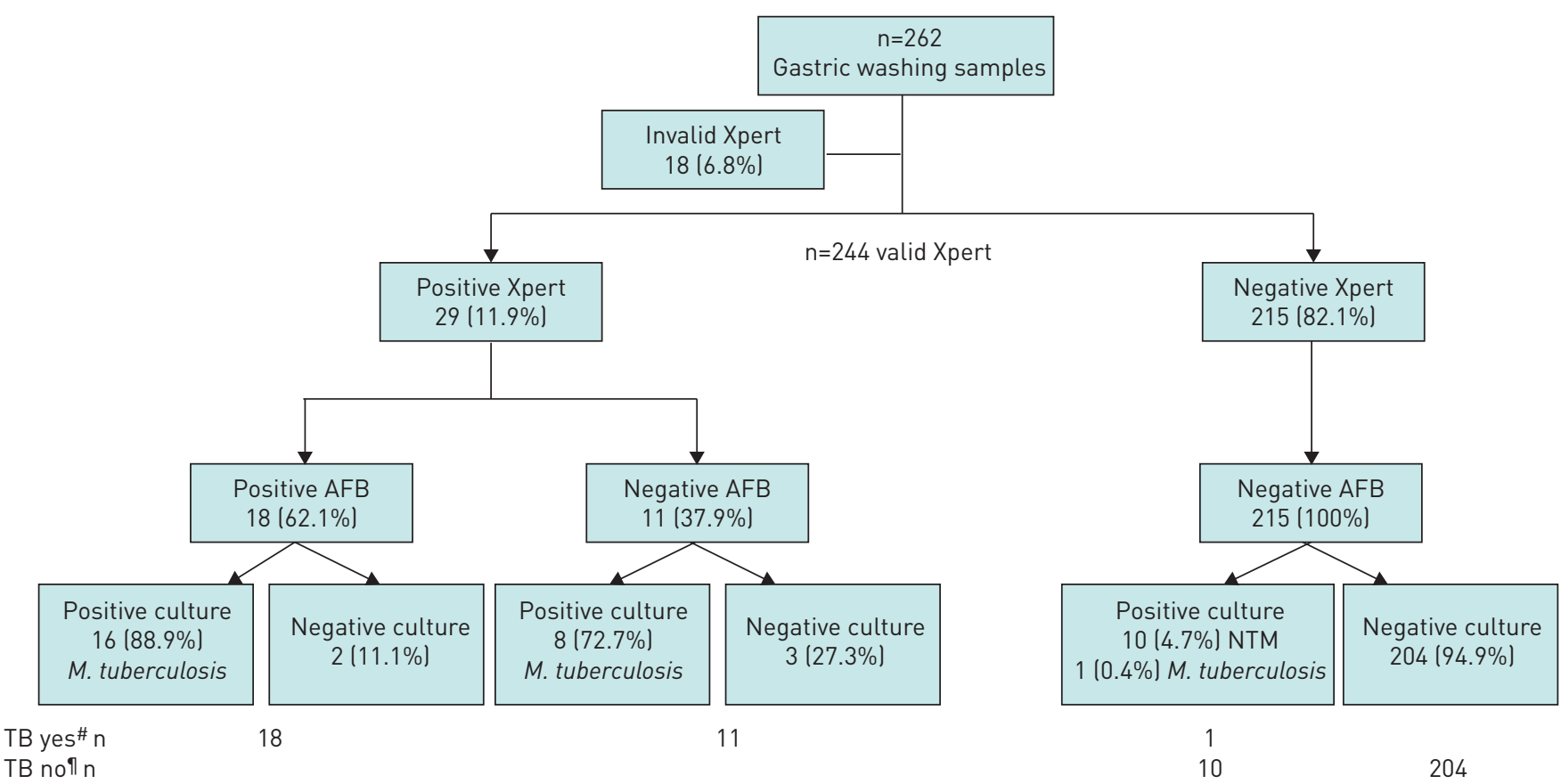

FIGURE 3 Xpert MTB/RIF results for 262 gastric washing specimens from patients suspected of pulmonary tuberculosis (TB). Xpert: Xpert MTB/ RIF; AFB: acid-fast bacilli; M. tuberculosis: Mycobacterium tuberculosis; NTM: nontuberculous Mycobacteria. "\#: confirmed pulmonary TB; ${ }^{\uparrow}$ : TB not confirmed. 
profile detected. No additional Xpert tests were positive for M. tuberculosis, so it was not possible to confirm rifampicin resistance by Xpert.

Phenotypic DST was available in 17 out of these 23 patients while GenoType MTBDRplus testing was performed in 20 out of 23 patients and WGS in 19 out of 23 patients.

Considering phenotypic DST and/or WGS as the standard reference for detection of rifampicin resistance, we compared the results from Xpert MTB/RIF in one or two clinical specimens, depending on availability. Regarding the overall concordance between Xpert and WGS results, 15 (78.9\%) out of 19 showed rifampicin resistance detected by both tests. Among 17 isolates with phenotypic DST results available, 16 (94.1\%) were concordant with Xpert results. Among nine isolates with one Xpert test detecting rifampicin resistance, one was a false-positive TB case, due to a probable contamination of the bronchoscope. Seven patients with one Xpert test also presented with phenotypic DST and/or WGS rifampicin resistance, while in one patient WGS failed to identify rifampicin resistance-causing mutations.

The individual results for Xpert, GenoType MTBDRplus, WGS and phenotypic DST are presented in table 2.

\section{Discussion}

There is still a lack of information about Xpert performance under programmatic conditions in Brazil despite its regular use in many high-burden cities since 2014 [5]. Recent studies in Brazil showed that the use of the Xpert MTB/RIF increased detection of TB cases among paucibacillary patients [14], adolescents [15] and the HIV-positive population [16].

The high diagnostic performance observed in this study has been reported in other studies by STEINGART et al. [17], Chang et al. [18], Li et al. [19] and Theron et al. [20]. Xpert MTB/RIF outperformed smear microscopy and established diagnosis in a significant proportion of patients who were smear-negative, detected additional culture-negative patients and has excellent rule-out value for MDR-TB. The pooled sensitivity and specificity obtained by CHANG et al. [18] in smear-negative specimens $(75.0 \%$ and $98.2 \%$, respectively) were lower than the rates detected in smear-positive specimens $(98.7 \%$ sensitivity and $98.2 \%$

TABLE 2 Rifampicin susceptibility profiles for rifampicin for 23 pulmonary Mycobacterium tuberculosis isolates comparing Xpert MTB/RIF, GenoType MTBDRplus, phenotypic drug susceptibility tests (pDST) and whole-genome sequencing (WGS) looking for mutations that confers resistance to rifampicin

\begin{tabular}{|c|c|c|c|c|c|c|c|}
\hline Sample & $\begin{array}{l}\text { Xpert tests } \\
\text { performed } n\end{array}$ & Sample & $\begin{array}{l}\text { Xpert } \\
\text { result }\end{array}$ & $\begin{array}{l}\text { GenoType } \\
\text { plus }\end{array}$ & pDST & $\begin{array}{l}\text { WGS: rpoB } \\
\text { mutation }\end{array}$ & $\begin{array}{l}\text { Concordance Xper } \\
\text { and pDST or WGS }\end{array}$ \\
\hline 1 & 1 & Sputum & $1 \mathrm{R}$ & $\mathrm{R}$ & $\mathrm{R}$ & NA & Yes \\
\hline 3 & 1 & Sputum & $1 \mathrm{R}$ & NA & NA & No mutation & No \\
\hline 4 & 1 & Sputum & $1 \mathrm{R}$ & $\mathrm{R}$ & $\mathrm{R}$ & S450L & Yes \\
\hline 5 & 1 & Sputum & $1 \mathrm{R}$ & NA & $\mathrm{R}$ & NA & Yes \\
\hline 6 & 1 & Sputum & $1 \mathrm{R}$ & $\mathrm{R}$ & $\mathrm{R}$ & S450L & Yes \\
\hline 9 & 1 & GW & $1 \mathrm{R}$ & $\mathrm{R}$ & $\mathrm{R}$ & D435 V/H445D & Yes \\
\hline 10 & 2 & Sputum & $2 \mathrm{R}$ & $\mathrm{R}$ & $\mathrm{R}$ & S450L & Yes \\
\hline 11 & 2 & Sputum & $2 \mathrm{R}$ & $\mathrm{R}$ & $\mathrm{R}$ & S450L & Yes \\
\hline 12 & 2 & Sputum & $2 \mathrm{R}$ & $\mathrm{S}$ & NA & L430P/H445N & Yes \\
\hline 13 & 2 & Sputum & $2 \mathrm{R}$ & $\mathrm{R}$ & $\mathrm{R}$ & S450L & Yes \\
\hline 14 & 2 & Sputum & $2 \mathrm{R}$ & $\mathrm{R}$ & $\mathrm{R}$ & S450L & Yes \\
\hline 20 & 2 & Sputum & $2 \mathrm{R}$ & $\mathrm{R}$ & $\mathrm{R}$ & No mutation & Yes \\
\hline 21 & 2 & Sputum & $2 \mathrm{R}$ & $\mathrm{R}$ & $\mathrm{R}$ & S450L & Yes \\
\hline 22 & 2 & BAL & $1 \mathrm{R} ; 1 \mathrm{~S}$ & $\mathrm{~S}$ & NA & No mutation & Yes \\
\hline 23 & 2 & GW & $1 \mathrm{R} ; 1 \mathrm{~S}$ & $\mathrm{~S}$ & NA & NA & NA \\
\hline
\end{tabular}


specificity). Li et al. [19] also reported a higher sensitivity in patients with positive pulmonary smears (97-99\%) than in patients with negative smear results (68-73\%).

AsLAm et al. [21] showed that gastric washing is an interesting alternative to investigate PTB in adults unable to expectorate good-quality sputum, with a high Xpert MTB/RIF sensitivity compared to smear and culture. In this study, M. tuberculosis was detected on Xpert testing in $91.7 \%$ of smear-positive samples (excluding scanty), $88.2 \%$ of scanty positives and $11.4 \%$ with negative smears. KHALIL and BUTT [22] and Lu et al. [23] evaluated the Xpert performance to diagnose PTB in BAL, showing high sensitivity in this clinical specimen. Lu et al. found $33.8 \%$ of smear-positive samples among the cases with Xpert-positive results and confirmed TB. The good performance of Xpert MTB/RIF on BAL and gastric washing might be particularly useful for populations such as children or patients living with HIV infection.

Our study detected Xpert MTB/RIF-positive results in 23 clinical specimens collected from patients who had previous confirmed TB (1-3 years prior), but without any evidence of active TB. Molecular techniques with high sensitivity are able to detect DNA from both live and dead $M$. tuberculosis. Thus, a positive Xpert MTB/RIF result does not always imply viable bacilli and should not be used to monitor response to treatment, treatment failure or relapse [24-26]. This is a very important information for the clinician who orders and makes decisions based on Xpert MTB/RIF results.

The sample quality may influence Xpert performance, as described by ACUÑA-VILLAORDUÑa et al. [26], who found that mucopurulent sputum samples were associated with increased yield of Xpert in Uganda.

In this study, the specificity of the Xpert MTB/RIF assay was not influenced by the presence of NTMs. During the study period, 136 (6.3\%) NTMs were cultivated among 2148 pulmonary specimens tested without a single positive Xpert MTB/RIF result. This is concordant with a recent systematic review, which only identified one Xpert-positive specimen among 180 specimens with NTM. This information is relevant and has the potential to guide clinicians, especially in HIV patients with pulmonary disease plus AFB-positive and Xpert MTB/RIF-negative results [27].

As described earlier, several studies have shown good diagnostic accuracy of Xpert for diagnosing TB, but data on long-term clinical outcomes have been disappointing, as shown by Monedero-Recuero [28] and Hanrahan et al. [29], who showed high rates of nondiagnostic results and lack on improvement in the number of patients starting TB therapy. Cost-effectiveness analysis of Xpert implementation in South Africa showed neither decreasing cost nor improvement in the number of patients starting treatment [30]. However, our data represent the performance of Xpert MTB/RIF in a tertiary hospital with 1000 beds where the results are immediately available (real-time) to the clinicians based on the electronic system, which make the connections between the Xpert machine and the electronic patient record.

PINTO et al. [31] estimated the incremental cost-effectiveness ratio of Xpert as a substitute for two AFB tests in the diagnosis of drug-susceptible TB and provided support for decision-makers in Brazil as well as other countries with a low prevalence of TB - financial benefit can potentially be expected if physicians rely more on a negative Xpert result. Consequently, the empirical treatment might be reduced.

Importantly, the findings from the current study correlate with previous work that demonstrated the ability of Xpert to support decisions to shorten airborne isolation for hospitalised patients with presumptive TB due to its high NPV. CowAn et al. [32] showed that Xpert MTB/RIF may reduce airborne infection isolation in hospitalised patients with presumptive PTB and is comparably sensitive, more specific and more cost-effective than smear microscopy. The sensitivity of one Xpert, two Xperts, two smears or three smears compared to culture in this work was $0.85,0.95,0.70$ and 0.80 , respectively. The cost-effectiveness analysis demonstrated that an Xpert test is the most cost-effective strategy.

Many studies found variable sensitivity and specificity values for the detection of rifampicin resistance. Moreover, other studies have described false-positive resistant cases, which is cumbersome for clinical decision-making, mainly in settings where the prevalence of rifampicin resistance is low [33-35].

STEINGART et al. [17] obtained $95 \%$ of pooled sensitivity and $98 \%$ of pooled specificity for detection of rifampicin resistance. The sensitivity and specificity for rifampicin resistance detection in CHANG et al.'s [18] meta-analysis were $94.1 \%$ and $97 \%$, respectively, while BoEHME et al. [34] found sensitivity of $94.4 \%$ and specificity of $98.3 \%$.

In our study, 19 (90.5\%) out of 21 patients showed concordance between rifampicin resistance detection in Xpert with phenotypic DST and/or WGS. The concordance between Xpert and Genotype MTBDRplus was high. Genotype MTBDRplus had high concordance with phenotypic DST and WGS.

There were two discordant patients. The first showed one rifampicin-resistant Xpert nonconfirmed with another test and WGS showed a pan-susceptible profile. The growth of the bacilli for DNA extraction 
could have selected for the rifampicin-susceptible clones. This result might be a false rifampicin-resistance Xpert not confirmed with a second test, as observed in isolates 22 and 23 (table 2). The second patient had two rifampicin-resistance Xpert results, but the phenotypic DST and WGS showed a pan-susceptible profile. For this discrepancy, possible explanations include erroneous phenotypic testing result, resistant minority bacterial populations that were not detected by sequencing, mechanisms of resistance unknown or laboratory labelling error [36].

Regarding false-positive resistance detection, Williamson et al. [33] found that the Xpert test incorrectly detected rifampicin resistance in $31 \%$ of the evaluated cases. Possible explanations proposed by this group were the presence of mutation in the analysed genomic region associated with low-level rifampicin resistance and the presence of silent mutations, which results in failure of the corresponding probe to hybridise. For example, a silent CAG (Gln) to CAA (Gln) mutation at codon 510 was found in one isolate evaluated in this study and resulted in failure of the corresponding Xpert probe $\mathrm{A}$ to hybridise. Consequently, false-positive rifampicin resistance was confirmed. The same phenomenon was described by other authors in relation to a TTC (Phe) to TTT (Phe) mutation at codon $514[37,38]$.

Incidence of silent mutations varies geographically; they might be very frequent in some regions [39]. However, VALim et al. [40] studied 82 rifampicin-resistant M. tuberculosis isolates in Brazil and did not find silent mutation in the $r p o B$ gene.

In contrast, there are mutations in the $r p o B$ gene which confer resistance to rifampicin, but are not detected by Xpert because they are located outside the 81-bp region, called rifampicin-resistance-determining region, which is the region evaluated by the commercial assays. In a previous study, we found one of these mutations ( $r p o B$ Val170Phe) in two isolates using WGS without rifampicin resistance detected by Xpert. The drug resistance was confirmed by phenotypic DST [41]. Similarly, ANDre et al. [42] identified the $r p o B$ Ile491Phe mutation in up to $30 \%$ of MDR-TB isolates in Swaziland.

The confidence of WGS in predicting phenotypic drug resistance is dependent on our knowledge of the association between phenotype and genotype. The accuracy of predicting resistance varies among different classes of drugs as well as different drugs from the same class. M. tuberculosis strains with a minimum inhibitory concentration very close to the critical concentration will flip-flop between resistant and susceptible, thereby impacting the predictive value of the mutation causing resistance $[43,44]$.

This study corroborates WHO requirement for at least two resistance Xpert results to define rifampicin resistance [3] and we add to that the need to verify epidemiological information and the patient's background. The history of previous $\mathrm{TB}$, treatment failure and known contact with drug-resistant $\mathrm{TB}$ patients should also be considered in those cases where it was not possible to test the second clinical specimen after the first resistant Xpert result, because clinicians still need to make an informed decision about the best treatment option for each patient.

The main limitation of this study lie in the phenotypic DST and WGS gaps and missing information among the Xpert-resistant and -susceptible isolates. Moreover, PTB cases included in this study were not classified according to disease severity, which could influence Xpert MTB/RIF performance.

The new generation of Xpert MTB-RIF, the Xpert Ultra, increases sensitivity in paucibacillary samples (smear-negative specimens) with a mild decrease in specificity $[45,46]$, and might be an improvement in PTB diagnosis in the near future in Brazil.

In conclusion, Xpert MTB/RIF is a quick and accurate diagnostic assay to diagnose PTB and can help clinicians to make better and informed therapeutic decisions for patients suspected of PTB in the context of a tertiary hospital and outpatient clinic. Regarding evaluation of rifampicin resistance, it is critical to follow the requirements to repeat resistant Xpert tests and perform confirmatory Genotype MTBDRplus, mainly in paucibacillary samples. Another reasonable approach is to consider epidemiological information before changing the patient's treatment.

Acknowledgements: The National Program for TB Control, Ministry of Health, provided the Xpert MTB/RIF tests for this study. The Program for TB Control, São Paulo Health State Secretariat, provided logistic support for the Xpert tests. The Central Mycobacteriology Lab at Adolfo Lutz Institute performed phenotypic drug susceptibility tests.

Conflict of interest: C.S. Feliciano reports grants from FAPESP during the conduct of the study. L.C.B. Menon has nothing to disclose. L. Anselmo has nothing to disclose. A. Dippenaar has nothing to disclose. R.M. Warren has nothing to disclose. W.A. Silva Jr reports grants from FAPESP during the conduct of the study. V.R. Bollela reports grants from FAPESP during the conduct of the study.

Support statement: Research funding was received from the Fundação de Amparo à Pesquisa do estado de São Paulo (protocol number: 15/13333-3), and Fundação de Apoio ao Ensino, Pesquisa e Assistência do Hospital das Clínicas da 
Faculdade de Medicina de Ribeirão Preto da Universidade de São Paulo. Funding information for this article has been deposited with the Crossref Funder Registry.

\section{References}

1 World Health Organization (WHO). Global Tuberculosis Report 2018. Geneva, WHO, 2018.

2 Machado D, Couto I, Viveiros M. Advances in the molecular diagnosis of tuberculosis: from probes to genomes. Infect Genet Evol 2018; pii: S1567-1348(18)30933-X.

3 World Health Organization (WHO). Policy Statement: Automated Real-Time Nucleic Acid Amplification Technology for Rapid and Simultaneous Detection of Tuberculosis and Rifampicin Resistance: Xpert MTB/RIF System. Geneva, WHO, 2011.

4 Kohli M, Schiller I, Dendukuri N, et al. Xpert ${ }^{\oplus}$ MTB/RIF assay for extrapulmonary tuberculosis and rifampicin resistance. Cochrane Database Syst Rev 2018; 8: CD012768.

5 Brazil Ministry of Health. Secretariat of Health Surveillance. Department of Surveillance of Communicable Diseases. Rede de Teste Rápido para Tuberculose no Brasil: primeiro ano da implantação [Rapid Test Network for Tuberculosis in Brazil: first year of implantation]. Brasília, 2015.

6 Brazil Ministry of Health. Secretariat of Health Surveillance. Department of Surveillance of Communicable Diseases. Manual de Recomendações para o Controle da Tuberculose no Brasil. [Manual of Recommendations for the Control of Tuberculosis in Brazill. 2nd Edn. Brasília, 2019.

7 Coll F, McNerney R, Preston MD, et al. Rapid determination of anti-tuberculosis drug resistance from whole-genome sequences. Genome Med 2015; 7: 51.

8 Ates LS, Dippenaar A, Ummels R, et al. Mutations in ppe38 block PE_PGRS secretion and increase virulence of Mycobacterium tuberculosis. Nat Microbiol 2018; 3: 181-188.

9 Bolger AM, Lohse M, Usadel B. Trimmomatic: a flexible trimmer for Illumina sequence data. Bioinformatics 2014 30: $2114-2120$

10 Li H, Durbin R. Fast and accurate short read alignment with Burrows-Wheeler transform. Bioinformatics 2009; 25: $1754-1760$

11 Ponstingl H, Ning Z. SMALT - a New Mapper for DNA Sequencing Reads. 2010. https://f1000research.com/ posters/327

12 McKenna A, Hanna M, Banks E, et al. The Genome Analysis Toolkit: a MapReduce framework for analyzing next-generation DNA sequencing data. Genome Res 2010; 20: 1297-1303.

13 Winglee K, Manson McGuire A, Maiga M, et al. Whole genome sequencing of Mycobacterium africanum strains from Mali provides insights into the mechanisms of geographic restriction. PLoS Negl Trop Dis 2016; 10: e0004332.

14 Casela M, Cerqueira SMA, Casela TO, et al. Rapid molecular test for tuberculosis: impact of its routine use at a referral hospital. J Bras Pneumol 2018; 44: 112-117.

15 Sieiro TLA, Aurílio RB, Soares ECC, et al. The role of the Xpert MTB/RIF assay among adolescents suspected of pulmonary tuberculosis in Rio de Janeiro, Brazil. Rev Soc Bras Med Trop 2018; 51: 234-236.

16 Silva DR, Sotgiu G, D’Ambrosio L, et al. Diagnostic performances of the Xpert MTB/RIF in Brazil. Respir Med 2018; 134: 12-15.

17 Steingart KR, Schiller I, Horne DJ, et al. Xpert ${ }^{\circ}$ MTB/RIF assay for pulmonary tuberculosis and rifampicin resistance in adults. Cochrane Database Syst Rev 2014; 1: CD009593.

18 Chang $\mathrm{K}, \mathrm{Lu} \mathrm{W}$, Wang J, et al. Rapid and effective diagnosis of tuberculosis and rifampicin resistance with Xpert MTB/RIF assay: a meta-analysis. J Infect 2012; 64: 580-588.

19 Li S, Liu B, Peng M, et al. Diagnostic accuracy of Xpert MTB/RIF for tuberculosis detection in different regions with different endemic burden: a systematic review and meta-analysis. PLoS One 2017; 12: e0180725.

20 Theron G, Peter J, van Zyl-Smit R, et al. Evaluation of the Xpert MTB/RIF assay for the diagnosis of pulmonary tuberculosis in a high HIV prevalence setting. Am J Respir Crit Care Med 2011; 184: 132-140.

21 Aslam W, Tahseen S, Schomotzer C, et al. Gastric specimens for diagnosing tuberculosis in adults unable to expectorate in Rawalpindi, Pakistan. Public Health Action 2017; 7: 141-146.

22 Khalil KF, Butt T. Diagnostic yield of bronchoalveolar lavage gene Xpert in smear-negative and sputum-scarce pulmonary tuberculosis. J Coll Physicians Surg Pak 2015; 25: 115-118.

$23 \mathrm{Lu} \mathrm{Y,} \mathrm{Zhu} \mathrm{Y,} \mathrm{Shen} \mathrm{N,} \mathrm{et} \mathrm{al.} \mathrm{Evaluating} \mathrm{the} \mathrm{diagnostic} \mathrm{accuracy} \mathrm{of} \mathrm{the} \mathrm{Xpert} \mathrm{MTB/RIF} \mathrm{assay} \mathrm{on} \mathrm{bronchoalveolar}$ lavage fluid: a retrospective study. Int J Infect Dis 2018; 71: 14-19.

24 Lawn SD, Mwaba P, Bates M, et al. Advances in tuberculosis diagnostics: the Xpert MTB/RIF assay and future prospects for a point-of-care test. Lancet Infect Dis 2013; 13: 349-361.

25 Kennedy N, Gillespie SH, Saruni AO, et al. Polymerase chain reaction for assessing treatment response in patients with pulmonary tuberculosis. I Infect Dis 1994; 170: 713-716.

26 Acuña-Villaorduña $\mathrm{C}$, Orikiriza $\mathrm{P}$, Nyehangane $\mathrm{D}$, et al. Effect of previous treatment and sputum quality on diagnostic accuracy of Xpert ${ }^{\circledR}$ MTB/RIF. Int J Tuberc Lung Dis 2017; 21: 389-397.

27 Agizew T, Basotli J, Alexander H, et al. Higher-than-expected prevalence of non-tuberculous mycobacteria in HIV setting in Botswana: implications for diagnostic algorithms using Xpert MTB/RIF assay. PLoS One 2017; 12 e0189981.

28 Monedero-Recuero I. Xpert implementation in challenging scenarios: a brand-new car running on the same bumpy old roads. Public Health Action 2018; 8: 1.

29 Hanrahan CF, Haguma P, Ochom E, et al. Implementation of Xpert MTB/RIF in Uganda: missed opportunities to improve diagnosis of tuberculosis. Open Forum Infect Dis 2016; 3: ofw068.

30 Vassall A, Siapka M, Foster N, et al. Cost-effectiveness of Xpert MTB/RIF for tuberculosis diagnosis in South Africa: a real-world cost analysis and economic evaluation. Lancet Glob Health 2017; 5: e710-e719.

31 Pinto M, Steffen RE, Cobelens F, et al. Cost-effectiveness of the Xpert ${ }^{\bullet}$ MTB/RIF assay for tuberculosis diagnosis in Brazil. Int J Tuberc Lung Dis 2016; 20: 611-618.

32 Cowan JF, Chandler AS, Kracen E, et al. Clinical impact and cost-effectiveness of Xpert MTB/RIF testing in hospitalized patients with presumptive pulmonary tuberculosis in the United States. Clin Infect Dis 2017; 64 482-489.

33 Williamson DA, Basu I, Bower J, et al. An evaluation of the Xpert MTB/RIF assay and detection of false-positive rifampicin resistance in Mycobacterium tuberculosis. Diagn Microbiol Infect Dis 2012; 74: 207-209. 
34 Boehme CC, Nabeta P, Hillemann D, et al. Rapid molecular detection of tuberculosis and rifampin resistance. $N$ Engl J Med 2010; 363: 1005-1015.

35 Van Rie A, Mellet K, John MA, et al. False-positive rifampicin resistance on Xpert ${ }^{\circledR}$ MTB/RIF: case report and clinical implications. Int J Tuberc Lung Dis 2012; 16: 206-208.

36 The CRyPTIC Consortium, 100,000 Genomes Project, Allix-Béguec C, Arandjelovic I, et al. Prediction of susceptibility to first-line tuberculosis drugs by DNA sequencing. N Engl J Med 2018; 379: 1403-1415.

37 Moure R, Martín R, Alcaide F. Silent mutation in rpoB detected from clinical samples with rifampin-susceptible Mycobacterium tuberculosis. J Clin Microbiol 2011; 49: 3722.

38 Alonso M, Palacios JJ, Herranz M, et al. Isolation of Mycobacterium tuberculosis strains with a silent mutation in rpoB leading to potential misassignment of resistance category. J Clin Microbiol 2011; 49: 2688-2690.

39 Behdani M, Rafatpanah H, Mohammadzadeh A, et al. Evaluation of sensitivity of molecular methods for detection of rifampin-resistant strains amongst drug-resistant Mycobacterium tuberculosis isolates. Arch Pediatr Infect Dis 2017; 5: e40580.

40 Valim AR, Rossetti ML, Ribeiro MO, et al. Mutations in the rpoB gene of multidrug-resistant Mycobacterium tuberculosis isolates from Brazil. J Clin Microbiol 2000; 38: 3119-3122.

41 Feliciano CS, Namburete EI, Rodrigues Plaça J, et al. Accuracy of whole genome sequencing versus phenotypic (MGIT) and commercial molecular tests for detection of drug-resistant Mycobacterium tuberculosis isolated from patients in Brazil and Mozambique. Tuberculosis 2018; 110: 59-67.

42 André E, Goeminne L, Colmant A, et al. Novel rapid PCR for the detection of Ile491Phe rpoB mutation of Mycobacterium tuberculosis, a rifampicin-resistance-conferring mutation undetected by commercial assays. Clin Microbiol Infect 2017; 23: 267.

43 Domínguez J, Boettger EC, Cirillo D, et al. Clinical implications of molecular drug resistance testing for Mycobacterium tuberculosis: a TBNET/RESIST-TB consensus statement. Int J Tuberc Lung Dis 2016; 20: 24-42.

44 Witney AA, Cosgrove CA, Arnold A, et al. Clinical use of whole genome sequencing for Mycobacterium tuberculosis. BMC Med 2016; 14: 46.

45 Dorman SE, Schumacher SG, Alland D, et al. Xpert MTB/RIF Ultra for detection of Mycobacterium tuberculosis and rifampicin resistance: a prospective multicentre diagnostic accuracy study. Lancet Infect Dis 2018; 18: 76-84.

46 Opota O, Zakham F, Mazza-Stalder J, et al. Added value of Xpert MTB/RIF Ultra for diagnosis of pulmonary tuberculosis in a low-prevalence setting. J Clin Microbiol 2019; 57: e01717-18. 